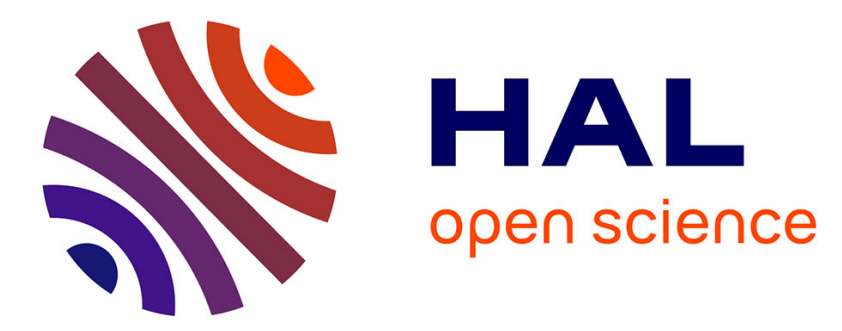

\title{
DET 2007 Special Issue: Horizontal and vertical integration of product data for the design of moulded interconnect devices
}

\author{
Yong Zhuo, Christian Alvarez, Klaus Feldmann
}

\section{- To cite this version:}

Yong Zhuo, Christian Alvarez, Klaus Feldmann. DET 2007 Special Issue: Horizontal and vertical integration of product data for the design of moulded interconnect devices. International Journal of Computer Integrated Manufacturing, 2009, 22 (11), pp.1024-1036. 10.1080/09511920902741091 . hal-00528090

\section{HAL Id: hal-00528090 \\ https://hal.science/hal-00528090}

Submitted on 21 Oct 2010

HAL is a multi-disciplinary open access archive for the deposit and dissemination of scientific research documents, whether they are published or not. The documents may come from teaching and research institutions in France or abroad, or from public or private research centers.
L'archive ouverte pluridisciplinaire HAL, est destinée au dépôt et à la diffusion de documents scientifiques de niveau recherche, publiés ou non, émanant des établissements d'enseignement et de recherche français ou étrangers, des laboratoires publics ou privés. 


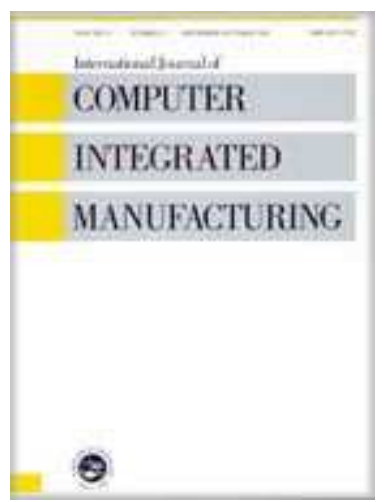

\section{DET 2007 Special Issue: Horizontal and vertical integration of product data for the design of moulded interconnect devices}

\begin{tabular}{|c|c|}
\hline Journal: & International Journal of Computer Integrated Manufacturing \\
\hline Manuscript ID: & TCIM-2008-IJCIM-0040.R2 \\
\hline Manuscript Type: & Special Issue Paper \\
\hline $\begin{array}{r}\text { Date Submitted by the } \\
\text { Author: }\end{array}$ & 30-Sep-2008 \\
\hline Complete List of Authors: & $\begin{array}{l}\text { Zhuo, Yong; Xiamen University, Department of Mechanical and } \\
\text { Electrical Engineering } \\
\text { Alvarez, Christian; University Erlangen-Nuremberg, Institute for } \\
\text { Manufacturing Automation and Production Systems } \\
\text { Feldmann, Klaus; University Erlangen-Nuremberg, Institute for } \\
\text { Manufacturing Automation and Production Systems }\end{array}$ \\
\hline Keywords: & CAD/CAM, DESIGN, MECHATRONICS \\
\hline Keywords (user): & Moulded Interconnect Devices, STEP Product Model \\
\hline
\end{tabular}

\section{S ScholaroNE" \\ Manuscript Central}




\title{
Horizontal and vertical integration of product data for the design of moulded interconnect devices
}

\author{
Y. ZHUO ${ }^{\mathrm{a}}$, C. ALVAREZ ${ }^{\mathrm{b}^{*}}$ AND K. FELDMANN ${ }^{\mathrm{b}}$ \\ ${ }^{\mathrm{a}}$ Department of Mechanical and Electrical Engineering, Xiamen University, Xiamen, China; \\ ${ }^{\mathrm{b}}$ Institute for Manufacturing Automation and Production Systems, University Erlangen-Nuremberg, Erlangen, Germany
}

\begin{abstract}
In this paper, a methodology for the vertical and the horizontal integration of product data fitted to the needs of Moulded Interconnect Devices (3D-MID) is presented. For that purpose, an integrated product model based on STEP and the feature technology is developed. The application objects and schemas of STEP AP210 are used and extended to support the horizontal collaboration between electronic and mechanical design. Special attention is paid to the description of the three-dimensional structure of the circuit carrier and the circuit traces running within it and on its surface. In addition, the integrated product model also supports the hierarchical collaboration between upstream MID design and downstream simulation und manufacturing. Based on this product model, an effective communication between MID design in MIDCAD and the simulation of the injection moulding process in MOLDFLOW and manufacturing utilizing MID-specific automatic placement equipment could be established.
\end{abstract}

Keywords: Product model; STEP; Integration; MID

\section{Introduction}

Recently, research has been actively conducted to develop prototype systems and methodologies to support collaborative CAD (Gausemeier et al, 2006; Li et al, 2005). A collaborative CAD system cannot be simply set up through equipping a standalone CAD system with IT and communication facilities. Due to the complexity of collaborative design activities and the specific characteristics and requirements of CAD systems, it needs some innovations or even fundamental changes in many aspects of CAD systems, such as infrastructure design, communication algorithms, geometric computing algorithms, product modelling, dynamic data sharing etc. (Noel and Brissaud, 2003).

Moulded interconnect devices (3D-MID) offer the possibility to combine mechanical and electronic functions in one single component. The enhanced design freedom and the integration of electronic as well as mechanical functions in a single injection-moulded part allow a substantial miniaturization. They provide enormous technical and economic potential and offer a remarkably improved ecological behaviour compared to conventional circuit boards (Feldmann, 1998). The mutual dependency between the geometry and the electronics makes it impossible to independently design one aspect without considering the other one in the system integration design stage (VDI 2206, 2004). An MID design process is distributed, parallel and integrated.

At the Institute for Manufacturing Automation and Production Systems (FAPS), an integrated system for the design of moulded interconnect devices has been developed (Feldmann et al, 2006; Zhuo et al, 2006). The basic elements of this so-called MIDCAD system are the functions 3D-placement and 3D-autorouting for the

${ }^{*}$ Corresponding author. Email: alvarez@faps.uni-erlangen.de 
definition of electronic components and the circuits on or within the 3D circuit carrier. In addition, MIDCAD contains a series of manufacturing-related functions, a 3D library with electronic components as well as a MID-specific feature library. As a result, MIDCAD can support the design of MID components more efficiently compared to other systems. Especially the development of an integrated, cross-domain MID product model within MIDCAD is expected to increase the efficiency.

In this paper, a methodology for the vertical and the horizontal integration of product data fitted to the needs of Moulded Interconnect Devices (3D-MID) is presented. The paper is structured as follows. Section 2 introduces the development of an integrated, cross-domain MID product model based on STEP and the feature technology. An effective communication between MIDCAD and the other applications to realize horizontal collaboration between electronic and mechanical design and to realize vertical collaboration between MID design, simulation and manufacturing, is described in section 3. Some concepts of the future work of the MIDCAD system are also described at the end of Section 3. Finally, section 4 draws a conclusion.

\section{Development of an integrated, cross-domain MID product model}

\subsection{Conception of an integrated, cross-domain MID product model}

The main problem within the design of MID products is the lack of an integrated product data model, which can take up all relevant ECAD/MCAD and CAE/CAM data and make it available to the corresponding applications in a standardized data format. Due to the absence of an integrated product data model the transfer of data from ECAD to MCAD and further on to manufacturing-related or simulation tools is carried out using specific data exchange files. The time needed for that process and the potential problems caused by transmission errors, redundancies and inconsistencies are not to be tolerated within an efficient development process.

In MID products, a large amount of electric, electronic and mechanical elements are being used, which are functionally linked in a very close way. Together they fulfil the specified overall requirements. Within the individual domains, specialized CAx systems are often used in different development stages. An optimal MID design is influenced by a number of factors. MID products directly integrate mechanical and electronic functions. Due to this special characteristic the traditional sharing of tasks during product development should be reconsidered. Mechanic and electronic as well as mould and tool designers must already work together closely in the concept phase. Therefore, apart from the horizontal integration of domain-specific information (e. g. from the fields of electronics and mechanics), the vertical integration of information coming from different product development stages (e. g. CAD/CAE/CAM) of an MID product inside a common, integrated product data model is also required, see figure 1 . Horizontal and vertical integration in a single product model build a basis to facilitate the organization of a collaborative design approach. In the vertical direction, upstream design and downstream manufacturing-related activities and in the horizontal direction, design teams from different disciplines are linked. That way, the complexity of collaborative design activities and the specific requirements of an MID design process can be addressed.

In order to describe such an integrated product data model, the relevant techniques such as multiple views (Bronsvoort and Noort, 2004), the feature technology, STEP and partial models or multi models (Xu and Wang, 2002) are being used. To describe the mechatronic products, the already existing application protocols (AP) of STEP have been reviewed regarding their ability to take up MID-specific product data. The APs in question were AP210 (ISO 10303-210, 2001), AP203 (ISO 10303-203, 2005), AP212 (ISO 10303-212, 2001) and AP214 (ISO 10303-214, 2003).

Figure 1. Concept of an integrated MID product model 
The developed integrated MID product model is based on several multi-disciplinary partial models. These domain-specific partial models describe an MID product in an object-oriented way and on the abstract level of the individual domains. The MID product model is built up as an aggregation of partial models and represents the actual relationship between the partial models of different fields.

The MID product model consists of two basic partial models: one for the description of the electric circuits and one for the 3D geometry of the circuit carrier. For the ECAD and MCAD partial models, two views are important: the functional view and the physical view. Using a functional model, electronic- and structurerelated aspects of MID products can be described in an abstract way. A physics model can be linked to the functional model. It is responsible for the description of the mechanics- and geometry-related data. This schema, which can also be found in STEP AP210, serves as a basis for the development of an integrated MID product model and the horizontal and vertical integration of product data. As certain properties of MID products cannot be represented by the partial models of AP210, an extension of the partial models needs to be taken into consideration.

The feature technology can also be used for description purposes and the vertical integration of CAE/CAM data within the integrated MID product model (VDI 2218, 2003). Via the feature technology, simulation-, manufacturing- and assembly-related data such as material properties or boundary conditions can be stored in the integrated product model already at the design stage. That way, the data can be used for further analysis, e. $\mathrm{g}$. for the simulation of the two shot moulding process.

\subsection{Horizontal integration of electronic and mechanical data}

The schema of AP210 is used as basis for the description of the horizontal integration of domain-specific data within the MID product model. The current status of AP210 makes it possible to realize a mechanic/electronic-integrated data model for the design of MID products.

For the description of the mechanic/electronic-integrated product data, a definition of the views for the fields of electronics and mechanics has to be given. In AP210, the data models from the functional view are responsible for the description of the electronic functionality, whereas the data models from the physical view are responsible for the description of the mechanic- and geometry-related information.

The functional and the physical view each have their own design view and usage view. The relevant application objects and the relationships between the functional and the physical view and between the design and the usage view respectively are presented in figure 2 .

Figure 2. Definition of the mechanic/electronic-integrated products (ISO 10303-210, 2001)

Using the MID technology, mechanical and electronic functions can be integrated directly within one single product. The description of the electronic and mechanic partial models and the product structure based on AP210 can be realized without major difficulties as there are corresponding application objects available in the AP210 schema. The challenge is rather to enhance the model of the circuit carrier including the circuits running on its surface to obtain a $3 \mathrm{D}$ representation. This enables the full description of the direct integration of electronics and mechanics in 3D MID technology in every detail.

\subsubsection{Description of the special structure of the circuit carrier}

In order to support the geometric design of the 3D circuit carrier, the corresponding data model has to describe the physical structure from the design view. A suitable application object in AP210 for that purpose is the application object Interconnect_module, which needs to be enhanced according to the special requirements of the structure of the $3 \mathrm{D}$ circuit carrier. As the $3 \mathrm{D}$ circuit carrier integrates the mechanical 
structure and the electronic functionality, it mainly consists of two parts: one part is of pure mechanic/geometric nature, the other part holds an electronic function in addition, see figure 3.

Figure 3. Definition of the special structure of the 3D circuit carrier

The geometric structures especially geared to describe the electronic functionality are called MID-specific features. They can be divided into two categories:

- Type I: A feature belonging to type I behaves like a normal electronic component, which is embedded within the circuit carrier. Examples are connectors, battery holders and vias. A new application object Component_feature which is linked to the AP210 object Bare_die_component is defined to enhance the description of this type of feature, see figure 4.

- Type II: This type contains a function which logically connects the electronic components placed on the circuit carrier and the special structures of type I. Examples for features of this type are the 3D circuits. In order to describe elements of type II, the AP210 object Stratum_feature has to be enhanced, see figure 5.

Even though circuit carriers based on MID technology only consist of one single component, the special MID structures discussed above are treated as separate components in the internal product data model and therefore the whole circuit carrier is modelled as an assembly in the design system. The structure of the assembly and the relationship between the application objects are shown in figure 4.

Figure 4. Definition of the internal assembly relationships of the circuit carrier

\subsubsection{Description of the structure of the $3 D$ circuits}

The specific characteristic of MID product is that the circuits do not run on a conventional 2D printed circuit board but directly on the surface or even inside a 3D circuit carrier. Therefore, the physical connectivity of each circuit belongs to one of the following three connectivity relationships, which in turn correspond to three AP210 application objects: Intra_stratum_join_relationship, Inter_stratum_join_relationship and Stratum_embedded_component_join_relationship (see figure 5 and figure 6).

- The first connectivity relationship (Intra_stratum_join_relationship) represents the connection between two circuit segments belonging to the same surface. In AP210, the term stratum normally stands for a layer of a multilayer PCB. Due to the special characteristics of MID products, stratum is extended in this context and regarded as the surface of the circuit carrier to describe the structure of a 3D circuit in MID products.

- The second connectivity relationship represents the connection between two separate segments running on two neighbouring surfaces which are connected by a point on the common edge of the two surfaces. The connection point is represented by the object Layer_connection_point, the relationship by the object Inter_stratum_join_relationship.

- The third connectivity relationship is the connection between a circuit segment and an MID-specific feature (Type I, e. g. connector or via) or an embedded electronic component. The object Stratum_embedded_component_join_relationship is used to represent this relationship. Therefore, the connection of two circuit segments by via can be represented by the third relationship. This is done by connecting two circuit segments separately with two connecting points belonging to the via on two surfaces.

Figure 5. Important Objects and their relationships for the definition of 3D circuits

The circuit segment on a surface corresponds to the object Conductor which implements the electronic connection between two points on the same surface. Conductor consists of the two objects Land and Conductive_interconnect_element. While the object Conductive_interconnect_element is extended to describe the geometric structure of a circuit segment between the connecting points, the object Land is used for the 
description of the connectivity between the connection points of the electronic component and of the circuit. The geometric shape of land depends on the type of the components. The shape of the circuits mainly depends on the result of the routing process and the cross-sectional form of the circuit. Therefore the additional attributes of the object Conductive_interconnect_element are added to describe the cross-sectional form, the trajectory and the points defined by the routing process.

Figure 6. Example for the description of 3D circuits

By using the objects mentioned above the elements of the physical connectivity of the circuits can be represented. The geometry of the circuits also has to be described in a detailed way within the data model. The geometric description of the circuit segments on a surface (Intra_stratum_join_relationship) forms the basis for the description of the whole circuit.

\subsection{Vertical integration of CAE- and CAM-information}

In order to realize the vertical integration of data from different product development phases, the feature technology is applied to describe the MID product model. That way, analysis-related data, e. g. material data or boundary conditions etc., can be assigned to the corresponding features, and later be used to create a simulation model for the injection moulding process.

\subsubsection{Definition of feature ontology for MID products}

Most of the 3D circuit carriers are thin-walled, thermoplastic and injection-moulded. Methodologies for the categorization and definition of the general features for such injection-moulded parts have already been presented in a larger number of scientific articles (e. g. Al-Ashaab et al, 2003 or Deng et al, 2002). Based on these publications, the general features for the description of the construction elements of the 3D circuit carrier have been defined hierarchically, see figure 7 .

The different types of the general MID features are presented in the following:

- Part feature: This feature does not represent a concrete geometric structure but the whole geometric model of a circuit carrier. It is an abstract feature describing the general information of the 3D circuit carrier. It is used especially to save important analysis- and manufacturing-related information, such as material properties or boundary conditions for the simulation of the injection moulding process.

- Wall feature: This feature represents the basic geometric structure, such as the thin walls of a thermoplastic circuit carrier.

- Development feature: The term development refers to the fact that this type of feature has been developed from the wall feature of the circuit carrier. Examples are hole, rib, boss, catch etc.

- Treatment feature: This type of feature refines the geometric structure by generation of chamfers, roundings, fillets etc. This type of feature is commonly supported in conventional CAD/CAM systems. However, it is necessary for the designer to define whether these features can be suppressed during CAE analysis. Thus they also contain analysis-related information.

Figure 7. Feature categories for MID circuit carriers

In contrast to the features containing only mechanical or geometric data such as wall, hole or rib, MIDspecific features are characterized by additionally supporting the integration of electronic functions. As described in section 2.2.1, the MID-specific features can be divided into the two types Component_feature and Stratum_feature. Figure 8 contains a few examples of MID-specific features.

The two types of MID-specific features are defined as follows: 
- Component feature: This type of feature corresponds to the application object Component_feature representing type $I$ of the special geometric structure such as connectors, vias or battery holders. Apart from geometry-related information, the MID-specific feature via e. g. contains the additional electronic information that there is a conductive connection between two surfaces. That way, this connection can be calculated in the 3D autorouting procedure.

- Stratum feature: This type of feature corresponds to the application object Stratum_feature, representing type II of the special geometric structure. It mainly comprises 3D circuits and lands. The MID-specific feature land corresponds to the object Land which describes the geometry of the connection between the circuit and the electronic component. The MID-specific feature $3 D$ circuit corresponds to the object Conductive_interconnect_element and describes the geometry of the 3D circuits.

Each MID feature has its corresponding geometry attributes. To support the parametric modelling the main dimensions and other important geometry parameters such as thickness are saved as the attributes of the MID features. The geometry of the features can easily be modified afterwards due to the use of a parametric modelling method. Apart from the attributes mentioned above, there also exists relationship information that specifies how features can be combined to form the design model. For example, the part feature has pointers to all the wall features, the wall feature has pointers to its embedded development, component and stratum features, and the treatment features have pointers to their parent features. In addition to the attribute information, the features also have certain behaviours including specification, creation, modification, removal and query of attributes, etc. Using an additional feature tree these general and MID-specific features are created, organized and operated by calling corresponding behaviours.

Figure 8. Examples of MID-specific features

\subsubsection{Application of features for the integration of CAE/CAM data}

The MID features developed for the MIDCAD system are not only simple form features consisting of structure-oriented groups of geometric elements. MID features rather contain geometric as well as nongeometric properties. The non-geometric properties can e. g. represent data from the fields of electronics, analysis, manufacturing, assembly etc.

The part features e. g. does not only contain the geometric attributes referring to the geometry model of the circuit carrier, but also analysis-related attributes for the flow simulation of the injection moulding process. These attributes can consist of material, constraints, boundary conditions, type of analysis and process parameters like the temperature of the mould and the cast, gate location or injection period. Using the feature wall, the analysis-related constraint regarding the gate location can be specified. Another specific analysisrelated attribute of features is the suppressibility. The suppressibility determines whether the geometry elements connected to a feature are negligible with respect to the mould simulation and can therefore be suppressed. This speeds up the automatic mesh generation for the simulation of the injection moulding process and reduces mesh errors at the same time.

Apart from analysis-related attributes, MID features can also contain assembly-related information for the subsequent development of the CAD/CAM-chain between MIDCAD and the MID placement machine. For example, the part feature contains the following attributes: type and key values of the placement machine, placement-related coordinate systems and a pointer to the model of the placement head.

Once the geometry of the carrier has been created, the general features can be defined by assigning the desired geometry to them. The geometry of the MID-specific features can be created and defined by using the developed feature behaviours based on the geometry of the carrier. After the geometric attributes have been defined, non-geometric attributes relating to these features can also be specified. With all the features created or defined and their relevant attributes specified, the features will be automatically merged into the integrated product model. 
Figure 9. Linking geometric and non-geometric information within MID-features

\section{Collaborative design between MIDCAD and other applications}

An integrated design process is necessary for moulded interconnect devices (Gausemeier and Frank, 2006). The results which have been achieved with the development of an integrated product model and the MIDCAD system form a good basis for the realization of an integrated design environment for the whole design process of moulded interconnect devices by combining tools from the areas of ECAD, MCAD, CAE and CAM and by establishing a link to external applications, see figure 10.

Within this context, the term external application refers to existing, separate applications to be used for MID development. The link between MIDCAD and the external application is realized by developing adequate interfaces based on the integrated product model. That way, the data generated by the external applications can be imported into and processed within MIDCAD. Furthermore, the interface also enables data access from outside.

Figure 10. An MID design environment based on the integration of CAx systems

\subsection{Horizontal collaboration between electronic and mechanical design}

MIDCAD is based on the commercially available 3D MCAD software Pro/ENGINEER and was developed using Pro/TOOLKIT. The geometric modelling and the subsequent mechanical design optimization can be carried out directly in MIDCAD or Pro/ENGINEER respectively. Consequently, no additional data transfers between both systems are necessary.

The 2D layout functions known from traditional ECAD systems are replaced by MIDCAD's own 3D layout functions. Even though a simple schematic design function was implemented in MIDCAD, an external 2D ECAD system remains responsible for this task in most of the cases. Hence, the data exchange between MIDCAD and ECAD is restricted to the information relevant for the schematic design phase. Thanks to a special interface, the components list and the net list exported from the ECAD system EAGLE can be imported into MIDCAD. As the data structure of the product model was developed in accordance with AP210, MIDCAD also offers a neutral interface. That way, the relevant electronic information generated following the AP210 standard from the ECAD system can be read by MIDCAD directly. At the same time the data of the schematic design that has been changed in MIDCAD can also be read by an ECAD system if it is equipped with an AP210 interface.

The basic data needed by MIDCAD are the 3D geometry data of the circuit carrier and the net and the component lists from the electronics design. The modelling of the original mechanical and geometric structure of the circuit carrier is done directly in Pro/ENGINEER. The net and component lists defined in ECAD are imported into MIDCAD using the specially developed interface. The special structure elements, which belong to MID-specific features (type I) such as vias etc, can be created by using MID-specific functions based on the original geometry model. That way the geometric structures needed to realize the electronic functions are created for the circuit carrier.

3D placement and 3D routing are two important steps in the course of MID design. First, the components are placed interactively. Afterwards, the routing is conducted - either manually or using the automatic 3D-routing functions. The 3D geometry of the circuits is then generated according to the definition of the circuits' crosssection and the result of the routing process. The position of the components can be changed manually at any time depending on the requirements of the routing itself or mechanical design requirements or according to the MID design and manufacturing rule checks. If necessary, the geometry of the circuit carrier can also be 
changed following the requirements of the routing process. The geometry of the circuits can also be changed depending on the electronic design requirement by running the autorouting function again. That way the horizontal collaboration between electronic and mechanical design can be realized.

\subsection{Hierarchical collaboration between MID design, simulation and manufacturing}

Apart from the link to the ECAD system mentioned before, a number of additional external applications can be linked to MIDCAD, e. g. for simulation purposes. The connection can be established in two different ways: by integration of the external application using OLE/COM or CORBA technologies or by using exchange files.

As most circuit carriers in MID technology are thermoplastic, either one- or two-shot-moulding is often used for manufacturing. By simulating the moulding process, mouldability and measures of quality can be assured in an early development phase. Even though Pro/ENGINEER already has an integrated module called "Pro/PlasticAdvisor" to simulate the moulding process, designers still need to manually generate an analysis model and to specify the analysis-related information. The analysis functions of Pro/PlasticAdvisor are also not suited to simulate the two-shot-moulding process. The integration of the Pro/PlasticAdvisor module in Pro/ENGINEER is in fact realized on a relatively low level. In order to provide an integrated environment for MID product design, the CAE-system "Moldflow Plastics Insight (MPI)" was directly connected to MIDCAD. A special interface has been developed based on the API of MPI and the OLE/COM-Technology, so that MIDCAD can automatically activate the analysis routines in MPI to run an injection moulding simulation, see figure 11 .

Figure 11. Collaboration between MID design and simulation of injection moulding process

To perform the simulation, the simulation model has to be built from the integrated product model. This involves the derivation of a simplified geometric model and the abstraction of non-geometric analysis-related information and boundary conditions, such as gate location etc. An intelligent module has been developed for that purpose. The module identifies the corresponding features and evaluates them with respect to the attribute suppressibility in order to hide the non-significant structure elements which are not necessary for the simulation. The abstraction of the non-geometric analysis information is mainly realized by querying the attributes of the part feature. As the analysis model is abstracted from the integrated product model and all the information is available for CAE analysis, the simulation can be activated without further interaction from the designer. After the simulation is finished, the results are analyzed and compared to predefined criteria, e. $\mathrm{g}$. maximum pressure. The criteria are provided as attributes of the part features in the integrated product model. If necessary, the process will go back to the design stage, where the relevant MID features are modified. Both the CAD geometry and the analysis-related data in the integrated model can be changed in order to obtain an improved structure and manufacturing result.

The development of suitable placement equipment and assembly systems for MID products is mainly affected by the complex geometry of the circuit carrier and the positions of electronic parts. Two alternative MIDspecific placement equipments were developed and realized at the institute FAPS (Krimi, 2001). The number of electronic components to be placed on the circuit carrier usually is so large that a manual input of the assembly-relevant data would be far too time consuming and too expensive. An automatic generation of manufacturing data and a corresponding interface to the placement equipment are therefore very important to improve design efficiency and product quality.

The integrated product model also serves as a basis for linking the MIDCAD system to a MID-specific placement equipment. The information relevant for the placement operation, e. g. the seating plane, the location of the sucking point, a simplified parametric model of the placement head and a placement-related 
coordinate system of the circuit carrier, can be integrated vertically or stored within the product model. Two placement-related modules have been developed in MIDCAD to establish a link to the placement equipment. The first module is responsible for the generation of the placement coordinates of the electronic components subject to their position on the circuit carrier and for the connection to the control system of the placement machine. The second module performs a static collision detection to identify potential problems during the placement process. Before creating the final collision-free instruction list, a kinematics simulation for dynamic collision checking during assembly can be applied. That way, a collision between the circuit carrier and the placement head can be detected in advance and measures to ensure a collision-free placement operation, such as replacing certain components and changing the dimensions of the carrier in the design stage or the placement sequence of the components, can be taken, see figure 12 .

Figure 12. Connection to MID placement equipment

\subsection{Potential collaboration in MID design}

\subsubsection{Data exchange between the MID expert system MIDIS and MIDCAD}

A re-use of the relevant data in MIDCAD from the description model of the MID expert system (Meier, 2002) is desirable. An automatic import of the data is to be preferred over a manual input in order to prevent errors resulting from a wrong data input and to assure the consistency of the data. For that, an appropriate interface between both systems has to be developed, so that the description model, which includes the data for a comprehensive description of the functionality of MID-applications, could be one subset and be integrated in the product model. The data in the corresponding information database of MIDIS, such as materials and parameters of process chains could be exchanged and managed by the PDM system.

\subsubsection{Derivation of data for laser structuring}

Another potential area of application is laser structuring. The circuits' geometry is created by the movement of the scanner and not by the geometry of the injection mould. Therefore, the derivation of the geometric data of the 3D-circuits is of great importance for the laser structuring process in order to generate the corresponding control programs. The appropriate method to link and integrate MIDCAD and production equipment for the laser structuring process must be developed. This includes the simulation of the laser structuring process. The MID technology database must be extended to include the information about the principles of 3D laser system and the specific requirements for laser structuring of MID product, such as the relationship between the width of the circuit and the relevant parameters of the laser. Furthermore, the Gerber file format, which is now commonly used between CAD and CAM systems in the electronics industry, must be examined and extended for the description of the 3D contour of circuit traces.

\subsubsection{Integrated PDM system and editor}

The integrated product model and the corresponding files can be managed by the integrated PDM system. That system handles the product data during the whole design process, i. e. data can be created, edited and deleted. The PDM system or the editor, which is developed based on the Windchill and PDMLink, can be started as an integrated module by selecting the corresponding menu item from the MIDCAD menu bar.

\section{Conclusion}

For the successful design of products based on the 3D-MID technology, it is important to establish a vertically seamless linkage between the upstream design and the downstream manufacturing processes. This can be 
achieved by creating intelligent strategies for effective information interchange and the horizontal interpersonal linkage of group work in the upstream design phases.

In this paper, a methodology for the vertical and the horizontal integration of product data fitted to the needs of moulded interconnect devices was presented. For that purpose, an integrated product model based on STEP and the feature technology was developed. The product model presented here was developed for an integrated collaborative design system for moulded interconnect devices. Based on this product model, an effective communication during the MID design process between MIDCAD, ECAD and MCAD and the simulation of the injection moulding process in MOLDFLOW and manufacturing utilizing MID-specific automatic placement equipment could be established. The integrated design environment increases the efficiency of the MID design process and also helps to overcome communication problems due to the usage of domain-specific terms. It is therefore a further target to integrate other MID relevant systems based on the integrated product model. This could be, for instance, the laser structuring system, kinematics and thermo simulations. Interoperability between MIDCAD and PDM system must be achieved.

\section{REFERENCES}

Al-Ashaab, A., Rodriguez, K., Molina, A., Aca, M.C., Saeed, M. and Abdalla, H., 2003. Internet-based Collaborative Design for an Injection-moulding System. Concurrent Engineering: Research and Application, 11, 289-297.

Bronsvoort, W.F. and Noort, A., 2004. Multiple-view feature modelling for integral product development. Computer-Aided Design, 34, 929-946.

Deng, Y.M., Britton, G.A., Lam, Y.C., Tor, S.B. and Ma, Y.S., 2004. Feature-based CAD-CAE integration model for injection-moulded product design. International Journal of Production Research, 40, 37373750 .

Feldmann, K., 1998. Introduction: MID - Entering Markets by Products and Technologies. In: Proc. of the 3rd International Congress on Molded Interconnect Devices. Erlangen, Germany, 5-17.

Feldmann, K., Zhuo, Y. and Alvarez, C., 2006. Entwicklung eines integrierten Konstruktionssystems für mechatronische Produkte mit räumlichen Schaltungsstrukturen (3D-MID). HNI-Verlagsschriftenreihe - Entwurf mechatronischer Systeme, 189, 371-383.

Gausemeier, J. and Frank, U., 2006. Stand und Perspektiven der Entwicklung mechatronischer Systeme. HNIVerlagsschriftenreihe - Entwurf mechatronischer Systeme, 189, 1-24.

Gausemeier, J., Hahn, A,. Kespohl, H.D. and Seifert, L., 2006. Vernetzte Produktentwicklung - Der erfolgreiche Weg zum Global Engineering Networking, Munich: Hanser.

ISO 10303-203, 2005. Industrial automation systems and integration - Product data representation and exchange, Part 203: Application protocol: Configuration controlled 3D designs of mechanical parts and assemblies. International Organization for Standardization, Geneva, Switzerland.

ISO 10303-210, 2001. Industrial automation system and integration - product data representation and exchange, Part 210: Application Protocol: Electronic assembly, interconnection, and packaging design. International Organization for Standardization, Geneva, Switzerland.

ISO 10303-212, 2001. Industrial automation systems and integration - Product data representation and exchange, Part 212: Application protocol: Electrotechnical design and installation. International Organization for Standardization, Geneva, Switzerland.

ISO 10303-214, 2003. Industrial automation systems and integration - Product data representation and exchange, Part 214: Application protocol: Core data for automotive mechanical design processes. International Organization for Standardization, Geneva, Switzerland.

Krimi, S., 2001. Analyse und Optimierung von Montagesystemen in der Elektronikproduktion, Thesis (PhD), FAPS, University Erlangen-Nuremberg. 
Li, W.D., Lu, W.F., Fuh, J.Y.H. and Wong, Y.S., 2005. Collaborative computer-aided desigñ Research and development status. Computer-Aided Design, 37, 931-940.

Meier, R., 2002. Strategien für einen produktorientierten Einsatz räumlicher spritzgegossener Schaltungsträger (3-D MID), Thesis (PhD), FAPS, University Erlangen-Nuremberg.

Noel, F. and Brissaud, D., 2003. Dynamic data sharing in a collaborative design. International Journal of Computer integrated manufacturing, 16, 546-556.

VDI 2206, 2004. Design methodology for mechatronic systems. Düsseldorf: VDI-Verlag.

VDI 2218, 2003. Information technology in product development Feature Technology. Düsseldorf: VDIVerlag.

$\mathrm{Xu}, \mathrm{X}$.Y. and Wang, Y.Y., 2002. Multi-model technology and its application in the integration of CAD/CAM/CAE. Journal of Materials Processing Technology, 129, 563-567.

Zhuo, Y., Alvarez, C. and Feldmann, K., 2006. An integrated design system for moulded interconnect devices (3D-MID). Proceedings of the 3rd CIRP Sponsored Conference on Digital Enterprise Technology, Setubal, Portugal. 


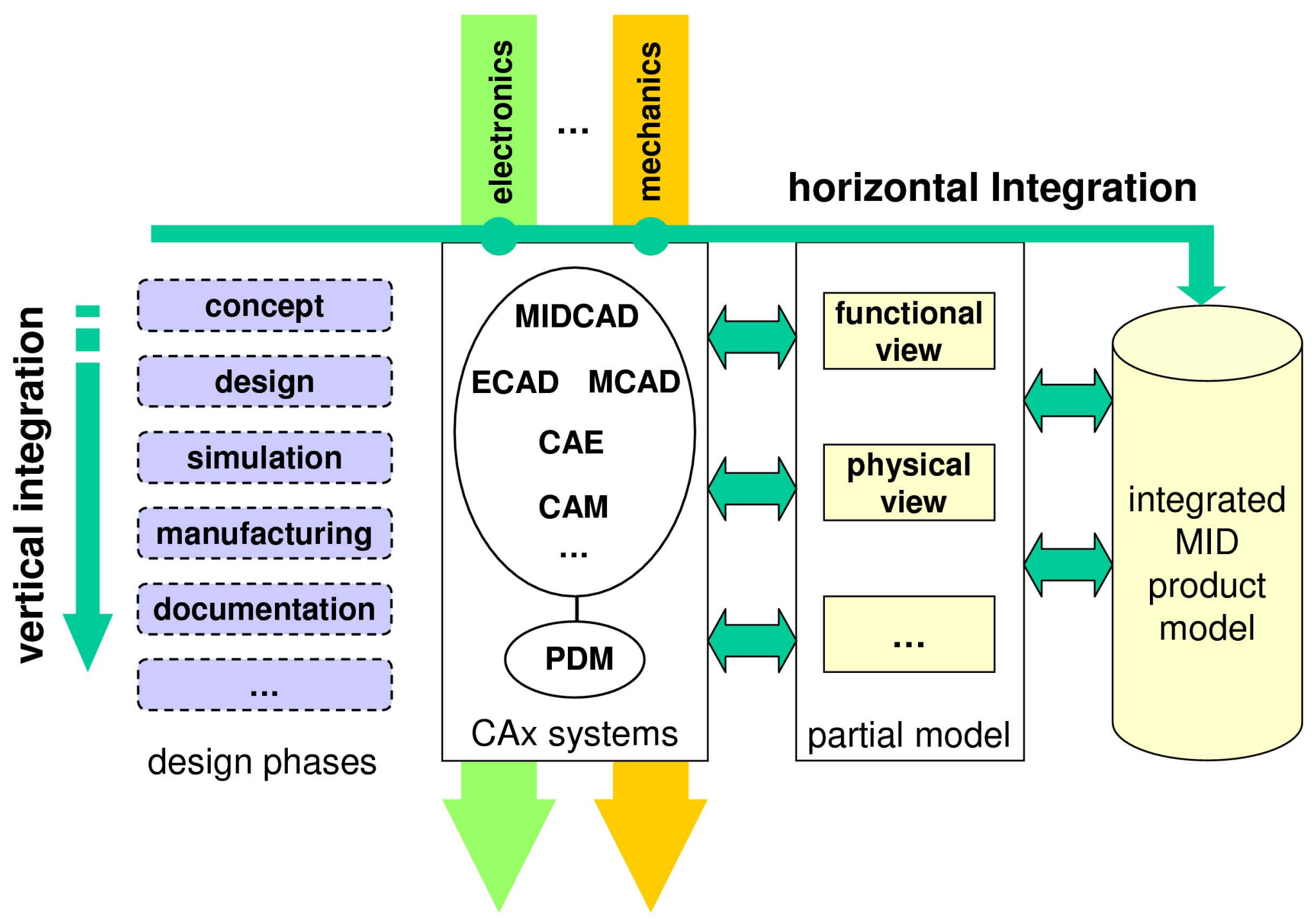

Figure 1. Concept of an integrated MID product model 


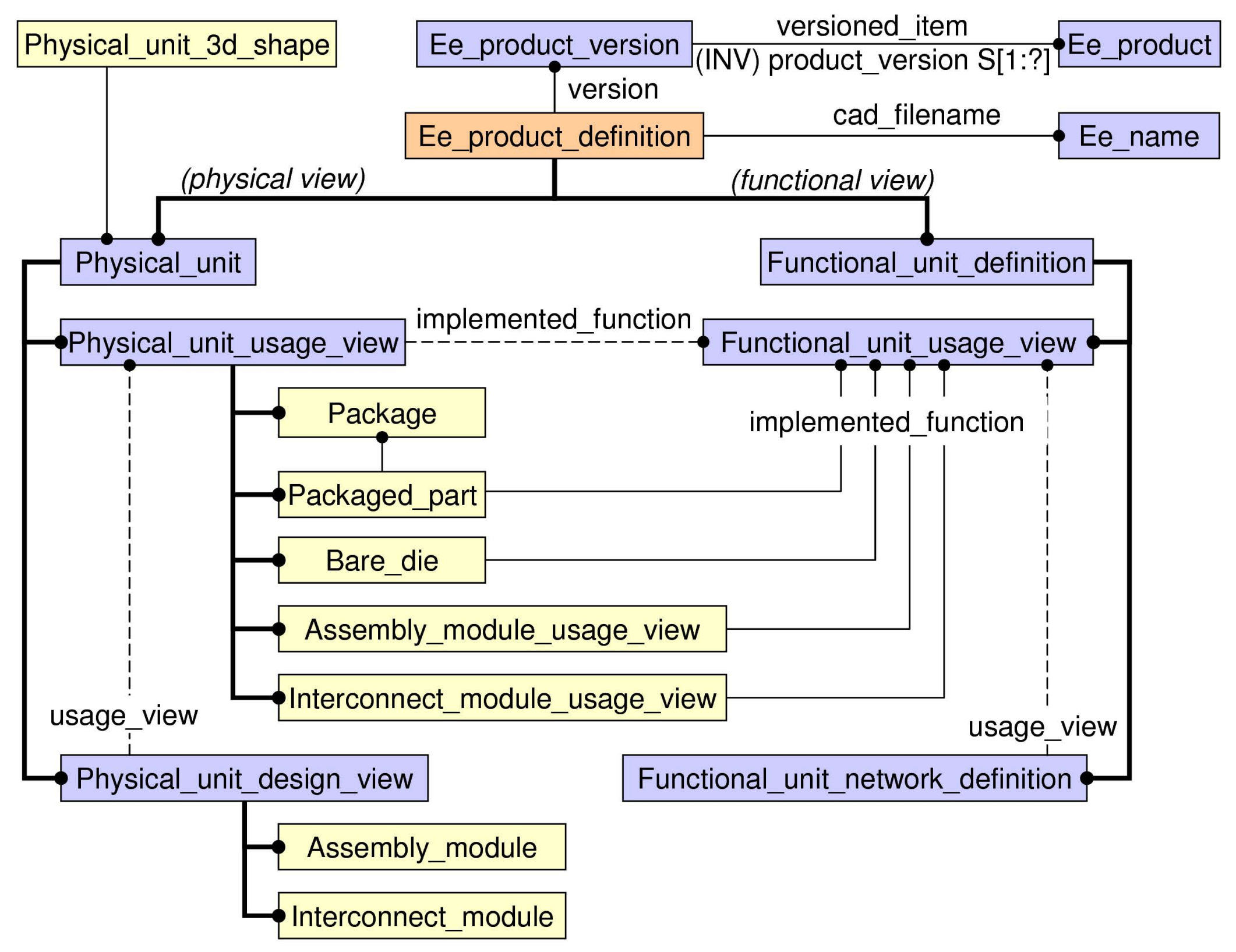

Figure 2. Definition of the mechanic/electronic-integrated products (ISO 10303-210, 2001) URL: http://mc.manuscriptcentral.com/tandf/tcim Email:ijcim@bath.ac.uk 


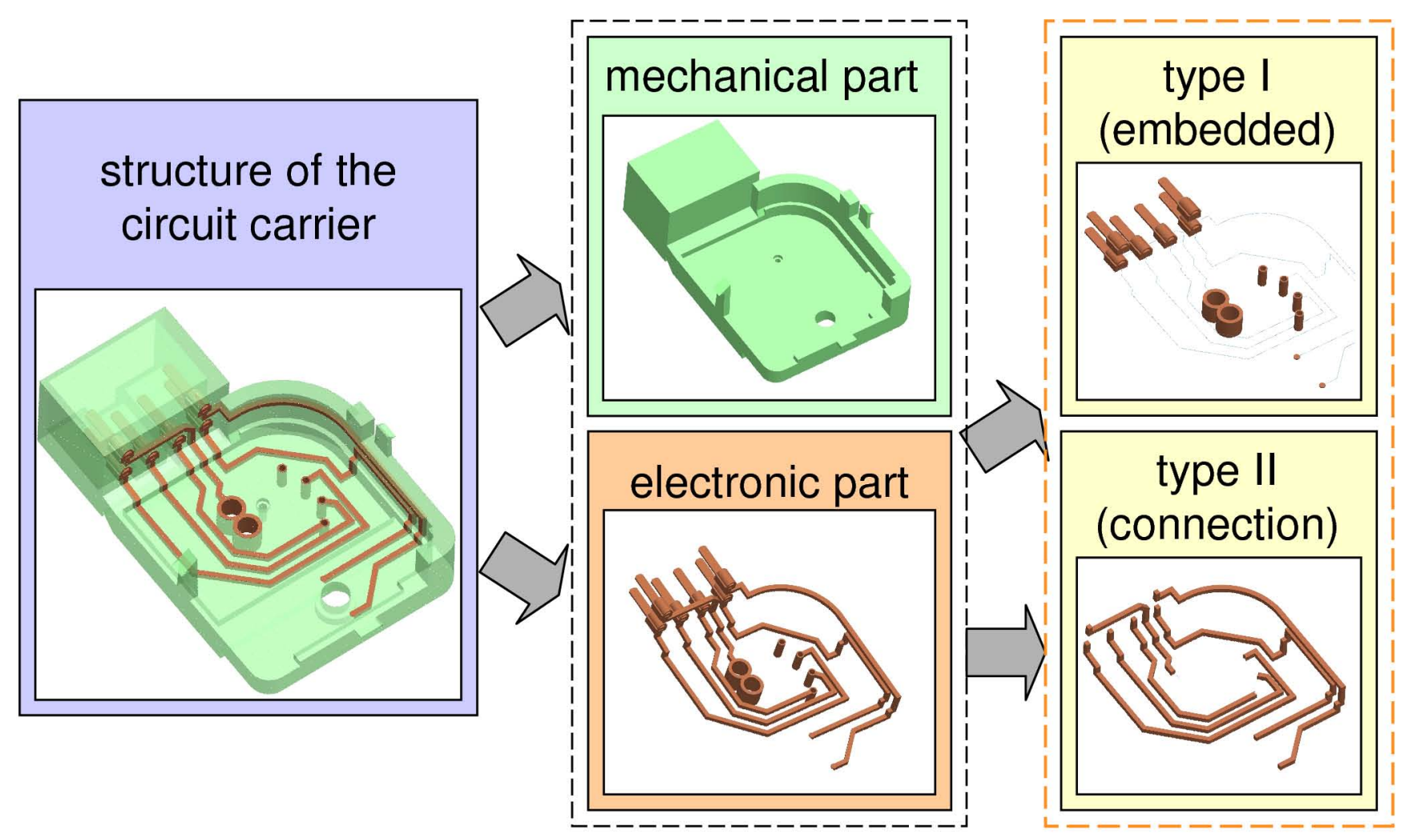

Figure 3. Definition of the special structure of the 3D circuit carrier 


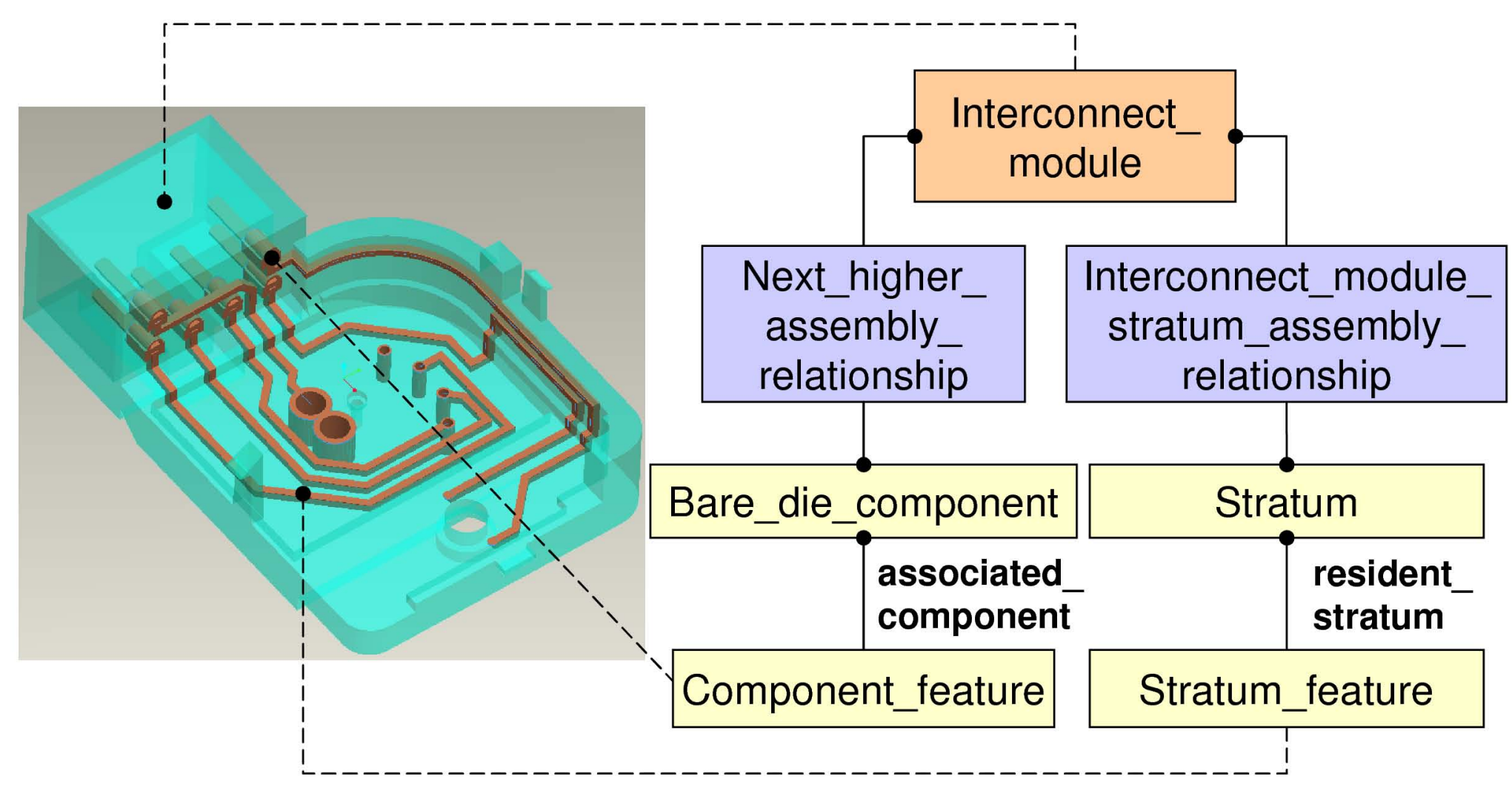

Figure 4. Definition of the internal assembly relationships of the circuit carrier 


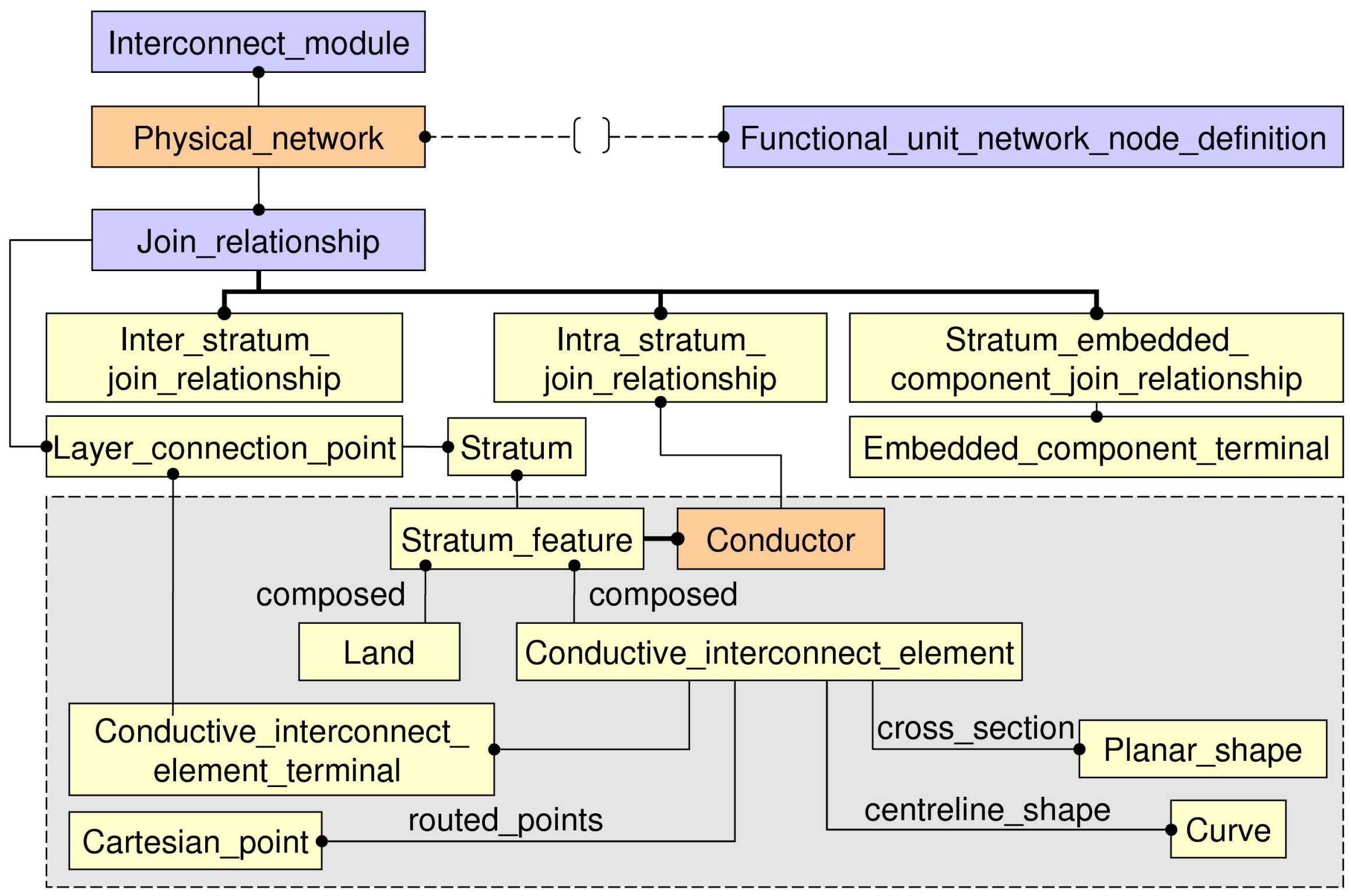

Figure 5. Important objects and their relationships for the definition of 3D circuits 


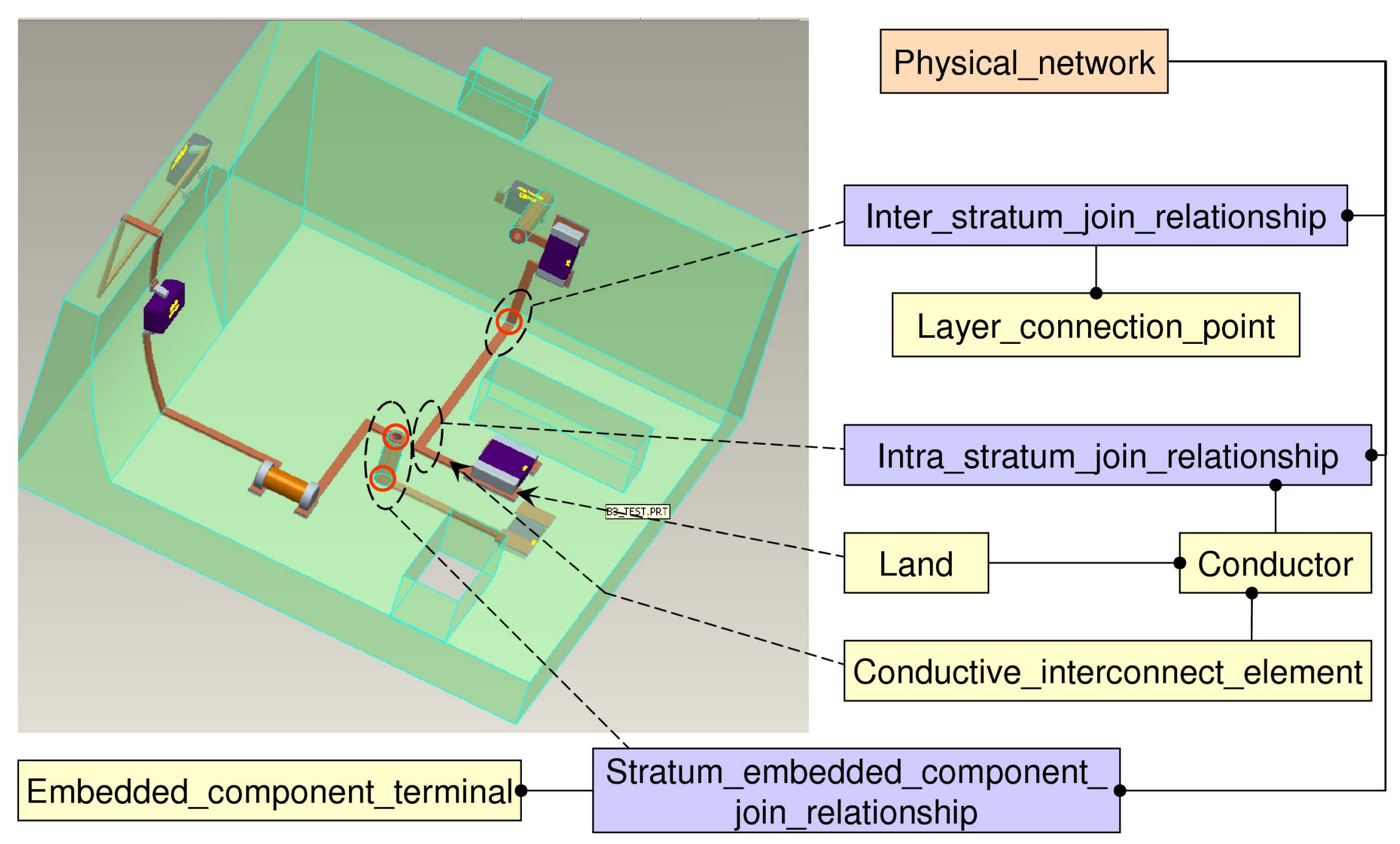

Figure 6. Example for the description of 3D circuits 


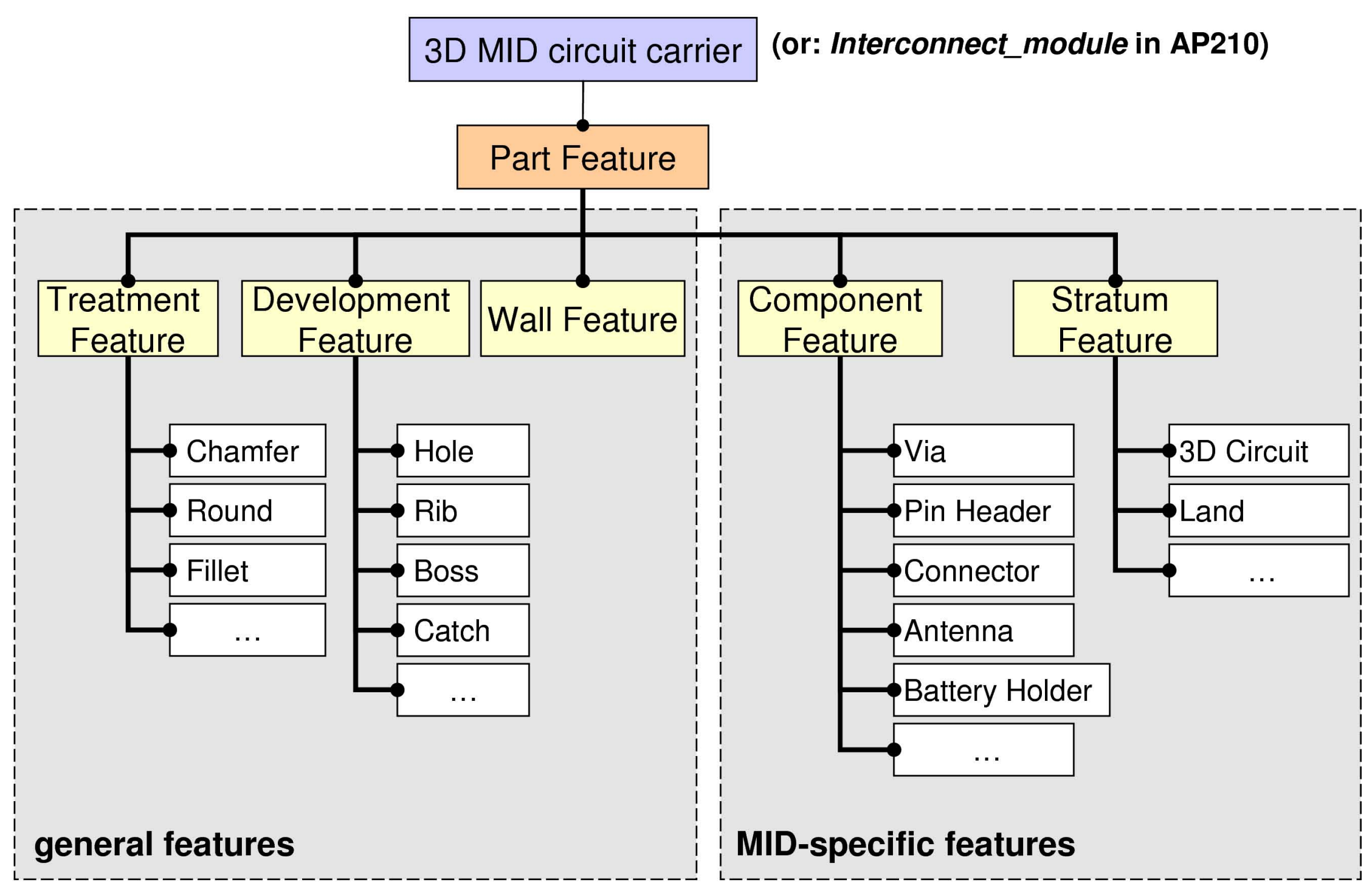

Figure 7. Feature categories for MID circuit carriers 


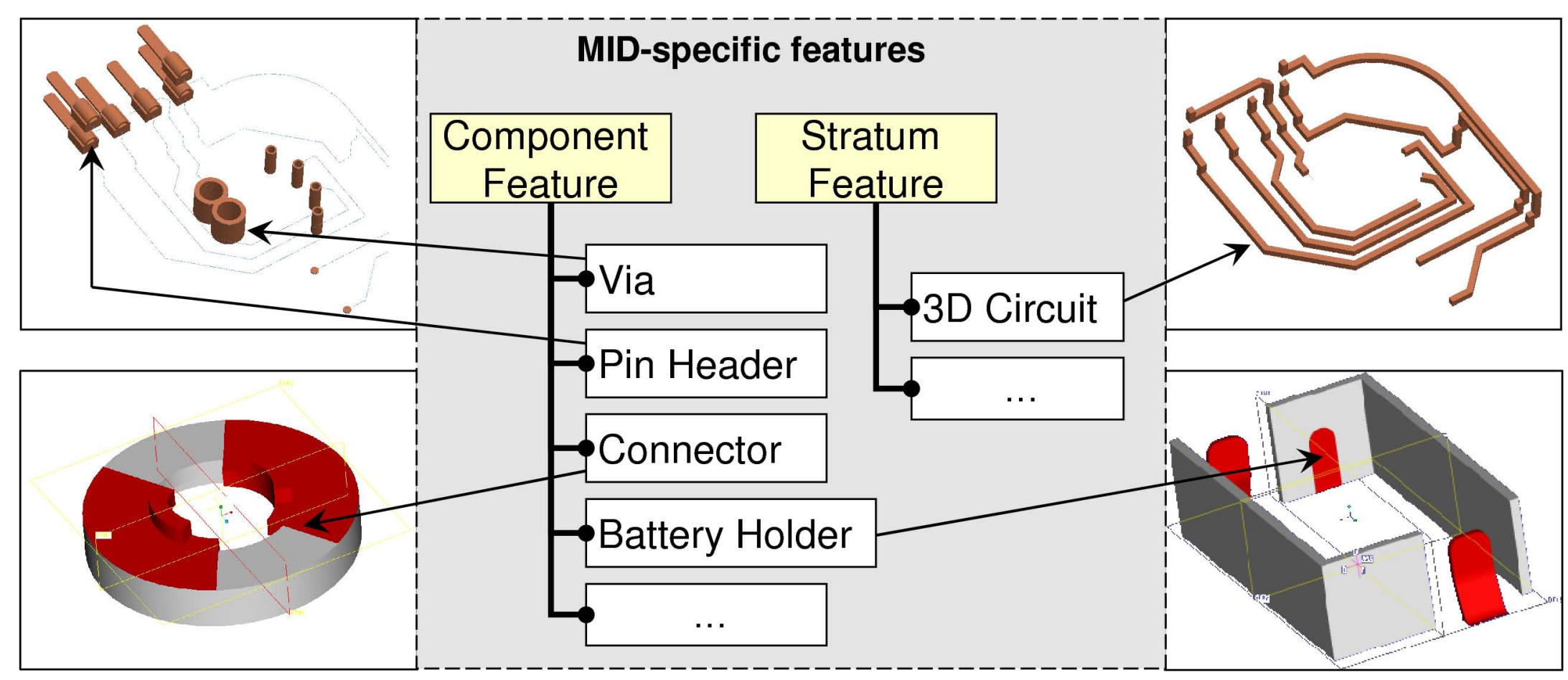

Figure 8. Examples of MID-specific features 


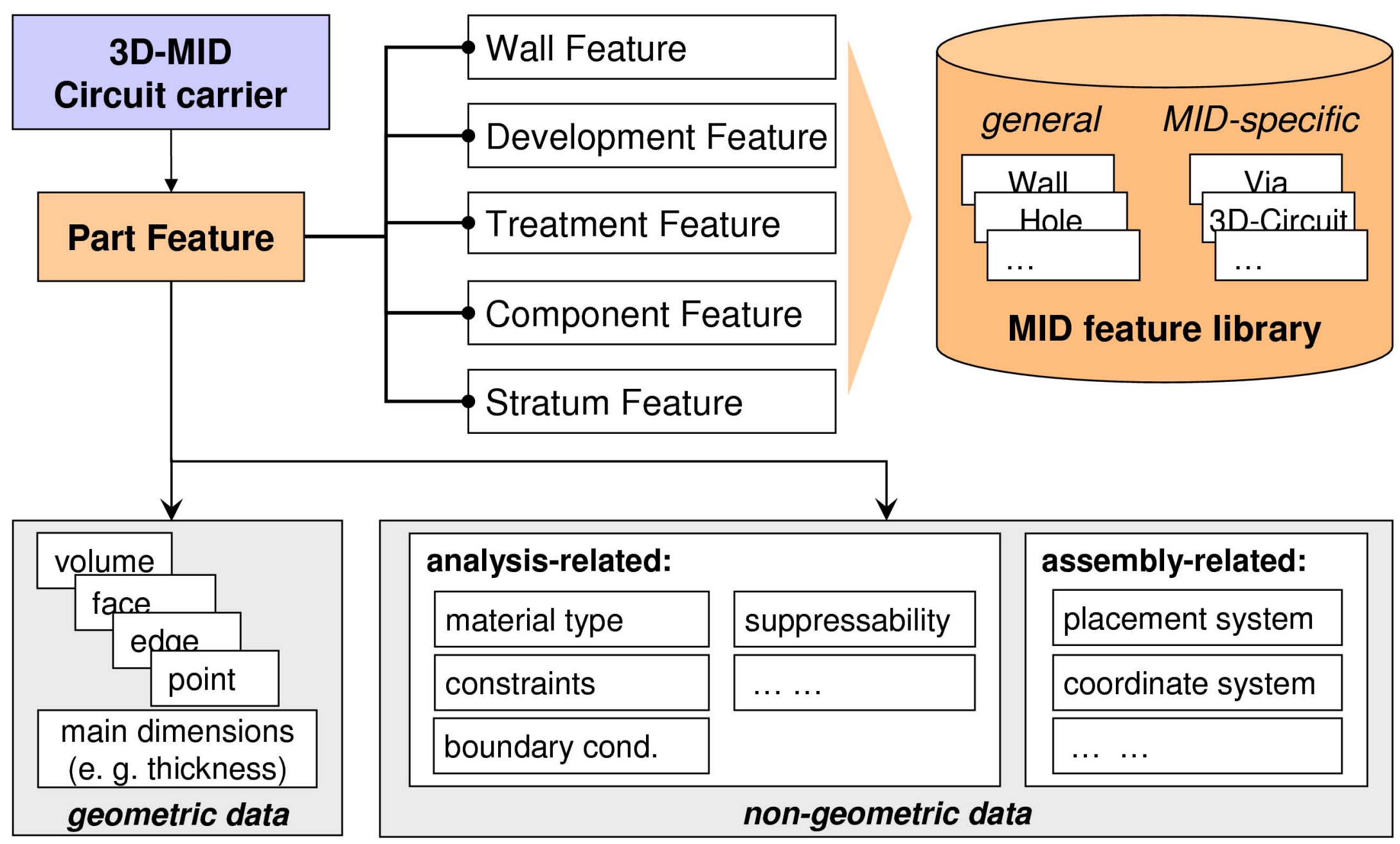

Figure 9. Linking geometric and non-geometric information within MID-features 


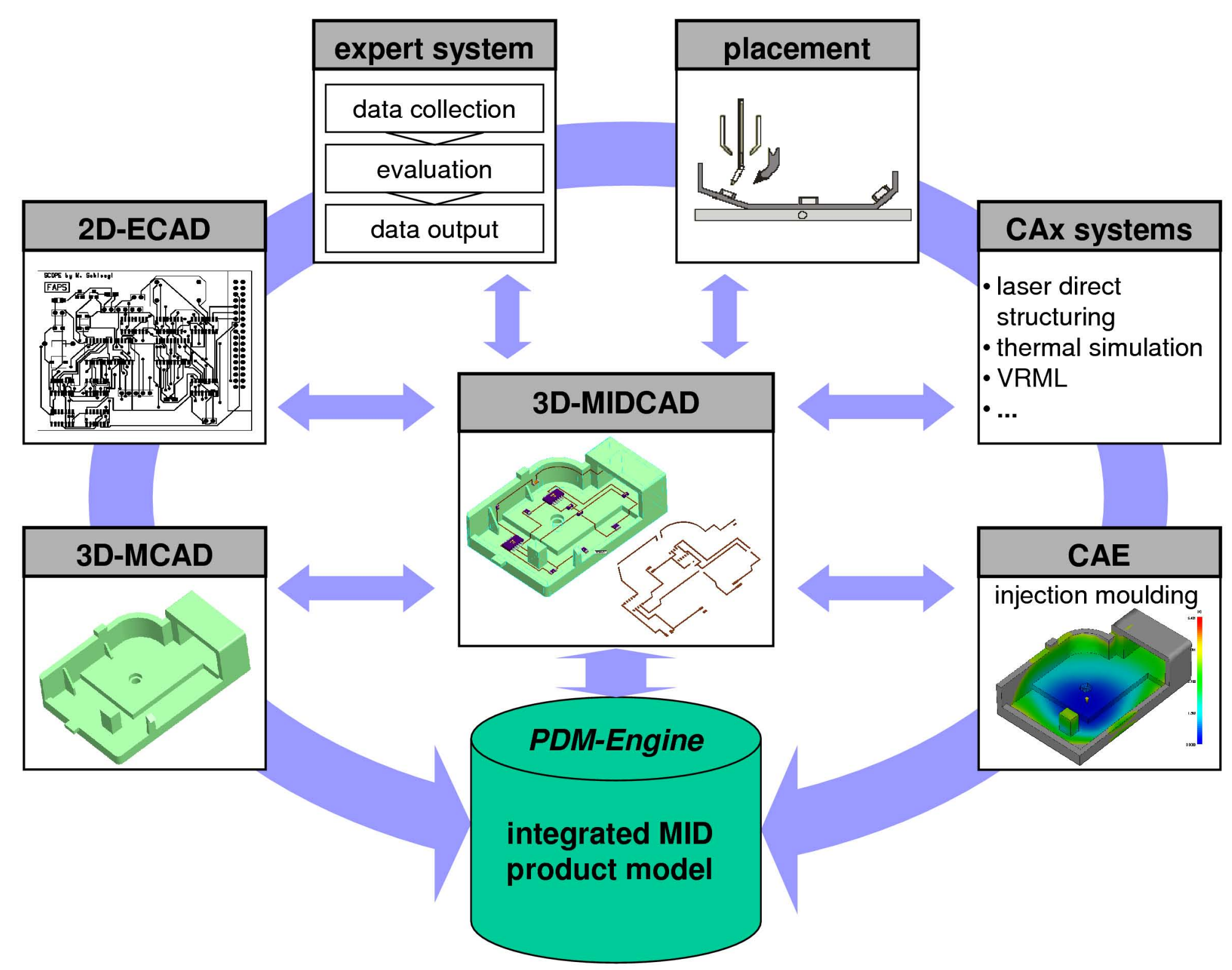

Figure 10. An MID design environment based on the integration of CAx systems 


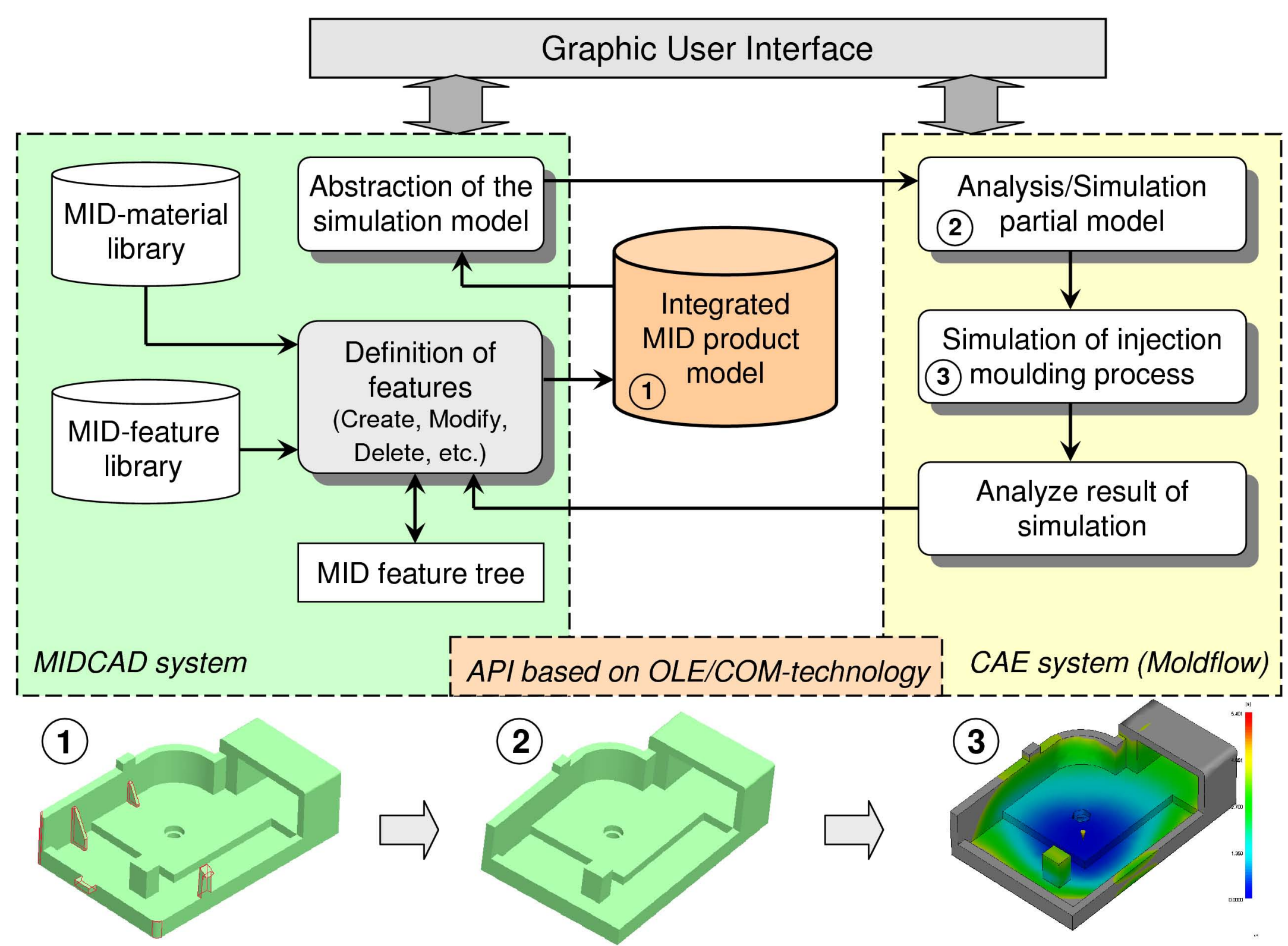

Figure 11. Collaboration between MID design and the simulation of the injection moulding process 


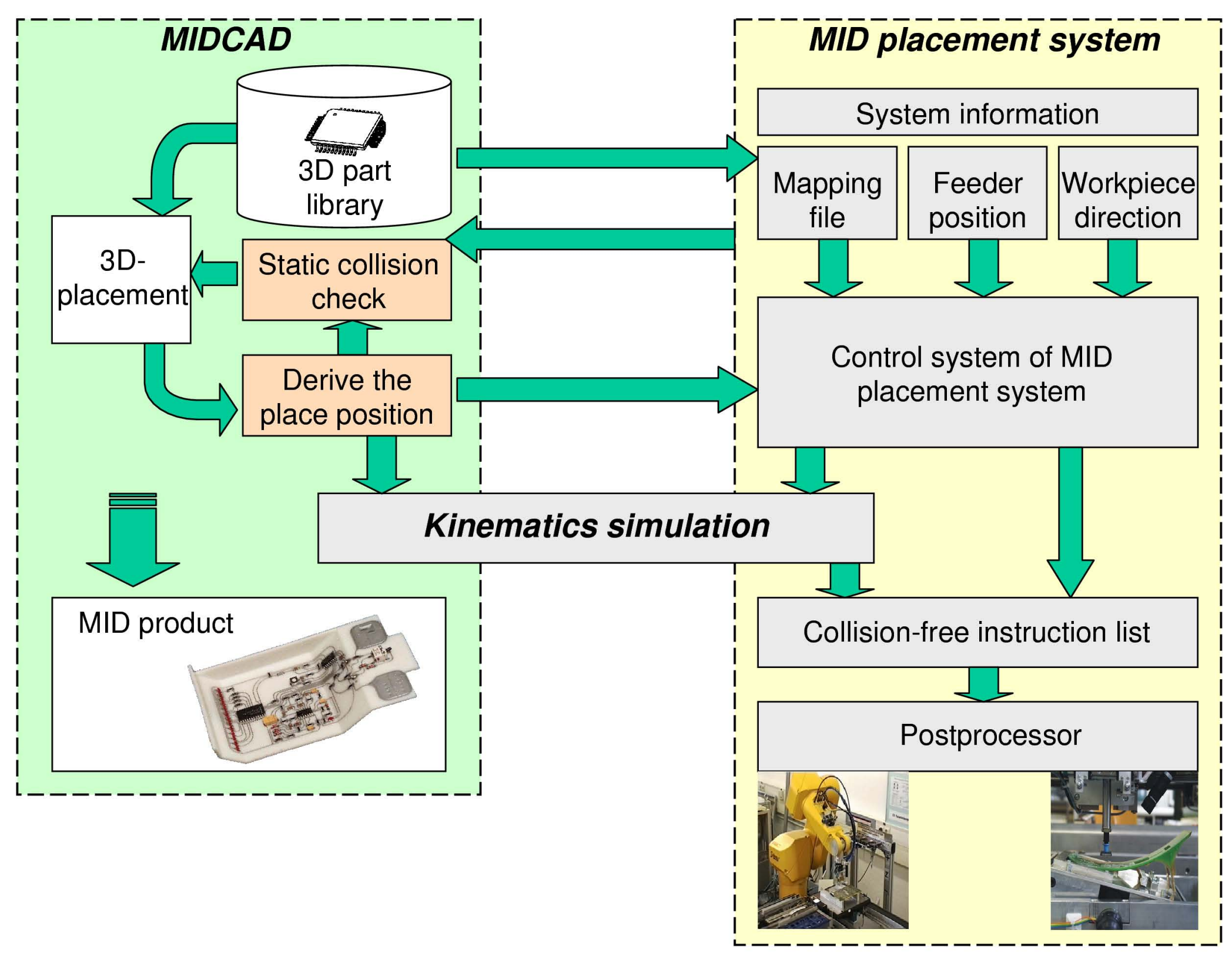

Figure 12. Connection to MID placement equipment 